\title{
Cathepsins B, L and cystatin $C$ in cyst fluid of ovarian tumors
}

\author{
Eva Kolwijck • Leon F. A. G. Massuger • \\ Chris M. G. Thomas · Paul N. Span · Marta Krasovec • \\ Janko Kos · Fred C. G. J. Sweep
}

Received: 23 October 2008 / Accepted: 19 October 2009 / Published online: 14 November 2009

(C) The Author(s) 2009. This article is published with open access at Springerlink.com

\begin{abstract}
Introduction In cancer, an extracellular and membrane bound localization of cathepsins contribute to the invasion of tumor cells at the basement membrane.

Methods This is the first study that explored levels of cathepsins B (CatB), L (CatL) and their inhibitor cystatin C (CysC) in the cystic fluid (CF) of ovarian tumors $(n=110)$. Results CF contained considerable amounts of CatB, CatL and CysC. Remarkable differences in $\mathrm{CatB}$ and $\mathrm{Cat} \mathrm{L}$ and $\mathrm{CysC} \mathrm{CF}$ levels were found between different histopathological tumor subtypes. Levels of CatB and CysC were significantly higher in $\mathrm{CF}$ of malignant serous tumors compared to those found in benign serous tumors $(p=0.010$ and $p=0.001$ respectively), whereas levels of CatL were
\end{abstract}

E. Kolwijck · L. F. A. G. Massuger

Department of Obstetrics and Gynecology,

Radboud University Nijmegen Medical Centre,

Nijmegen, The Netherlands

C. M. G. Thomas · P. N. Span · F. C. G. J. Sweep

Department of Chemical Endocrinology,

Radboud University Nijmegen Medical Centre,

Nijmegen, The Netherlands

P. N. Span ( $ه)$

Department of Radiation Oncology,

Radboud University Nijmegen Medical Centre,

P.O. Box 9101, 6500 HB Nijmegen, The Netherlands

e-mail: P.Span@rther.umcn.nl

M. Krasovec · J. Kos

Faculty of Pharmacy,

University of Ljubljana, Ljubljana, Slovenia

M. Krasovec · J. Kos

Department of Biotechnology,

Jožef Stefan Institute, Ljubljana, Slovenia significantly higher in CF of malignant mucinous tumors compared to those found in benign mucinous tumors $(p=0.035)$. CatB and CysC showed a strong correlation in the group of patients with malignant serous tumors ( $p<0.001 ; R=0.921$ ) suggesting that the increase in CatB might be balanced by a corresponding increase in CysC.

Conclusion Further studies are warranted to investigate cathepsins as possible prognostic biomarkers for the aggressiveness of ovarian cancer.

Keywords Ovarian cancer - Cathepsin - Cystatin - Cyst fluid - Ovarian tumor

\section{Introduction}

Epithelial ovarian cancer (EOC) is the most lethal gynecological malignancy worldwide. Because of the absence of early symptoms, approximately $70 \%$ of the patients are diagnosed with FIGO stage III or IV, when tumor cells already have metastasized to the upper abdomen (Colombo et al. 2006). Compared to other types of cancer, insight into the carcinogenesis and progression of ovarian cancer is limited by the lack of a well-defined precursor lesion and by the lack of data from early-stage cancers (Landen et al. 2008).

In neoplastic transformation, the epithelial surface first becomes multicell layered. Tumor cells then degrade the basement membrane and invade the stroma or become detached from the primary tumors and metastasize (CapoChichi et al. 2002; Scully 1995; Feeley and Wells 2001; Aznavoorian et al. 1993). It is now widely believed that the degradation of the basement membrane is catalyzed by proteolytic enzymes (Liotta et al. 1980; Turk et al. 2000; Duffy 1996). Among these enzymes are the lysosomal cysteine 
proteinases or cathepsins, which have an important physiological function in regulation of intracellular protein metabolism. The human family of cathepsins has 11 members (cathepsin B, C, F, H, K, L, O, S, V, W and X), which share a conserved active site that is formed by cysteine, histidine and asparagine residues. In cancer tissue, an increased expression of cathepsins has been described and an extracellular and membrane-bound localization of particularly cathepsin B (CatB) and cathepsin L (CatL) has been demonstrated, which might contribute to the invasion of tumor cells at the basement membrane (Turk et al. 2000; Kos et al. 2000; Premzl et al. 2006).

Numerous reports have now been published investigating cancer tissue expression of cathepsins (Duffy 1996; Schwartz 1995; Sloane et al. 1990). Although quantitation of cathepsins in extracellular fluid has several advantages compared to measurement in tissue, reports investigating extracellular cathepsin levels are rather limited (Kos et al. 2000). Higher levels of CatB and CatL were reported in sera of patients with several types of cancer (Gabrijelcic et al. 1992; Kos et al. 1997, 1998; Leto et al. 1997a; Strojan et al. 2001; Zore et al. 2001; Bhuvarahamurthy and Govindasamy 1995), including ovarian cancer (Nishida et al. 1995; Warwas et al. 1997), compared to levels in healthy subjects or patients with benign lesions. The possibility of measuring cathepsins in blood increases its clinical value as a tumor marker, but reported levels in the serum are significantly lower than levels in tumor cells of cancer patients (Kos et al. 2000). Lah et al. (1992) have shown that CatB was present in ascites of patients with ovarian carcinoma. Moreover, they showed that extracellular CatB was inhibited most strongly by cystatin $\mathrm{C}$ (CysC), which is an extracellular cysteine proteinase inhibitor (Lah et al. 1992). Recent studies on tissue of breast and ovarian cancer and melanoma showed that an imbalance of $\mathrm{CatB} / \mathrm{CysC}$ (zonder levels) was correlated with a more aggressive tumor behavior (Kos et al. 1997; Nishikawa et al. 2004; Yano et al. 2001).

Most ovarian tumors exhibit, sometimes multiple, cysts that can be large. The cyst fluid (CF) is in close contact with the tumor tissue. To our knowledge, no studies have been performed to investigate the levels of cathepsins or their endogenous inhibitors in this ovarian CF. In the present study, we explored CatB, CatL as well as CysC levels in $\mathrm{CF}$ of patients with benign, borderline and malignant ovarian tumors.

\section{Materials and methods}

Patients and cyst fluid collection

From our biobank containing ovarian cyst fluid (CF) samples of patients with ovarian tumors who underwent primary surgery at the Radboud University Nijmegen Medical Center in the period between 1988 and 1998, small portions of all $110 \mathrm{CF}$ samples were retrieved. Samples in this biobank were collected by aseptic fine needle aspiration at the department of pathology immediately after surgical removal of the tumor. After cooled transport to the laboratory, the $\mathrm{CF}$ samples were centrifuged at $3,000 \times \mathrm{g}$ for $10 \mathrm{~min}$ and the supernatant was stored at $-35^{\circ} \mathrm{C}$ in small portions until use. Histopathological diagnosis was performed by an experienced gynecologic pathologist and revealed 74 benign, 26 malignant and 10 borderline epithelial ovarian tumors. Informed consent was obtained from all participants.

\section{ELISA procedures}

For the quantitative analysis of CatB, CatL and CysC in ovarian $\mathrm{CF}$, commercially available specific ELISAs (sandwich ELISAs, Krka d.d., Novo mesto, Slovenia) were used, as developed by Kos et al. (1997) at Jožef Stefan Institute, Ljubljana, Slovenia. The components were purified and characterized, and the tests were optimized and performed as described (Kos et al. 1995). To define the optimal dilution, CF samples were serially diluted to the levels encompassing the range of the assays. The linearity of the response was evaluated by comparing the measured values with the calibration curves. The recovery was tested by the addition of different amounts of the antigen to the samples with known antigen concentration that varied from 88 to $108 \%$, comparing expected versus observed concentrations. The intra-assay coefficient of variance varied from 7.6 to $12.7 \%$. CF samples were diluted $1: 4$ for CatB and CatL and 1:100 for CysC prior to being applied to the wells of microtiter plate. A microplate reader (SLT Rainbow, Salzburg, Austria) was used to measure absorbance. Determination of the levels of cathepsins and CysC was carried out without prior knowledge of the histological or clinical outcome.

Parameters for aggressiveness of the tumor

From the pathology and medical reports of the patients with EOC, the following parameters were retrieved: histopathologic subtype and histopathologic grade, age at diagnosis and FIGO stage of disease. Histopathologic subtype and tumor grade were classified according to the World Health Organization (WHO) criteria (Servov et al. (1973)). Staging was performed using the International Federation of Gynaecologists and Obstetricians (FIGO) criteria (Pecorelli et al. 1999). In case of uncertainty about the primary location of the tumor, extensive immunohistochemical staining was performed. Only patients with confirmed primary EOC were included. 
Statistical analyses

Values are shown as median with range $(\mathrm{ng} / \mathrm{ml})$. Normality of distributions was analyzed by Kolmogorov-Smirnov testing. Data regarding CatB, CatL and CysC could be normalized by Ln transformation. Differences between two groups were tested by Student's $t$ test, and for more than two groups by ANOVA and subsequent post hoc Tukey's HSD tests. Correlations were assessed by Pearson correlation tests of the Ln-transformed values. A priori significance was set at a two-sided $P<0.05$. All statistical analyses were performed using SPSS version 16.0.01 (SPSS Inc. Chicago IL, USA).

\section{Results}

The mean age at diagnosis was 40 years $(\mathrm{SD} \pm 14)$ for patients with benign ovarian tumors $(n=74), 42$ years $(\mathrm{SD} \pm 14)$ for patients with borderline ovarian tumors $(n=10)$ and 57 years $(\mathrm{SD} \pm 11)$ for patients with EOC $(n=26)$. The mean age of patients with ovarian cancer was significantly higher than that of patients with benign $(p<0.001$, Student's $t$ test $)$ and borderline tumors ( $p=0.009$, Student's $t$ test). No differences in mean age were found between patients with benign and borderline tumors ( $p=0.898$, Student's $t$ test).

\section{Histopathological subtype}

Table 1 shows the median (range) concentrations of CatB, CatL and CysC in ovarian $\mathrm{CF}$ of patients with malignant, borderline and benign ovarian tumors, grouped by histological subtype. Within the benign group, CF levels of CatL were significantly different between serous cystadenomas and mucinous cystadenomas $(p=0.003)$ and between endometriosis cysts and mucinous cystadenomas $(p<0.001)$. Although generally not significantly different from the other benign histological subtypes, the median concentrations of $\mathrm{CatB}$ and $\mathrm{CatL}$ in cystic endometriosis were remarkably high and comparable to those found in $\mathrm{CF}$ of malignant tumors. CysC concentrations were in the same range for all benign subclasses. Within the group of borderline and malignant tumors, $\mathrm{CF}$ levels of $\mathrm{CatB}, \mathrm{CatL}$ and CysC did not differ significantly between histopathological subtypes.

Table 2 and Fig. 1 show median (range) CF levels of $\mathrm{CatB}, \mathrm{CatL}$ and CysC in benign (cystadenoma), borderline and malignant (cystadenocarcinoma) ovarian tumors according to the histopathological subtype. In serous

Table 1 Median levels (range) of cathepsin B, L and cystatin C (ng/ml) in ovarian tumor cyst fluid $(n=110)$ grouped by histopathological subtypes

\begin{tabular}{|c|c|c|c|c|}
\hline Histology & $N(\%)$ & Cathepsin B & Cathepsin L & Cystatin $\mathrm{C}$ \\
\hline \multicolumn{5}{|l|}{ Benign } \\
\hline Serous cystadenoma & $29(39)$ & $12(1-269)$ & $31(9-121)^{\mathrm{a}}$ & $572(131-1,781)$ \\
\hline Mucinous cystadenoma & $21(28)$ & $16(1-227)$ & $11(1-95)^{\mathrm{a}, \mathrm{b}}$ & $578(39-9,984)$ \\
\hline Dermoid cyst & $5(7)$ & $11(1-108)$ & $10(2-97)$ & $618(117-876)$ \\
\hline Cystic endometriosis & $16(22)$ & $71(1-177)$ & $69(8-233)^{\mathrm{b}}$ & $338(92-1,931)$ \\
\hline Corpus luteum cyst & $3(4)$ & $18(6-98)$ & $15(15-133)$ & $644(520-1,805)$ \\
\hline \multicolumn{5}{|l|}{ Borderline } \\
\hline Serous borderline tumor & $2(20)$ & $14(8-21)$ & $28(18-38)$ & $1,449(1,125-1,772)$ \\
\hline Mucinous borderline tumor & $6(60)$ & $31(2-118)$ & $75(8-166)$ & $697(474-3,897)$ \\
\hline Mixed-type borderline tumor* & $1(10)$ & 227 & 92 & 340 \\
\hline Unknown & $1(10)$ & & & \\
\hline \multicolumn{5}{|l|}{ Malignant } \\
\hline Serous cystadenocarcinoma & $10(38)$ & $65(3-269)$ & $61(8-163)$ & $1,443(448-5,972)$ \\
\hline Mucinous cystadenocarcinoma & $8(31)$ & $33(5-269)$ & $31(13-187)$ & $959(399-9,984)$ \\
\hline Endometrioid cystadenocarcinoma & $6(23)$ & $85(1-269)$ & $71(14-181)$ & $1,200(64-2,907)$ \\
\hline Mixed-type cystadenocarcinoma* & $1(4)$ & 95 & 67 & 1,232 \\
\hline Undifferentiated cystadenocarcinoma* & $1(4)$ & 110 & 75 & 109 \\
\hline
\end{tabular}

* A single concentration is listed. Statistical significance according to ANOVA $(p=0.001)$ with Tukey's HSD post hoc test of values normalized after Ln transformation

a $p=0.003$ for mucinous cystadenomas compared to serous cystadenomas

${ }^{\mathrm{b}} p<0.001$ for mucinous cystadenomas compared to cystic endometriosis 
Table 2 Median levels (range) of cathepsin B, L and cystatin C (ng/ml) in ovarian tumor cyst fluid $(n=67)$ of benign, borderline and malignant tumors grouped by serous and mucinous subtype

\begin{tabular}{|c|c|c|c|c|c|c|c|}
\hline Subtype & $N(\%)$ & Cathepsin B & $p$-value ${ }^{*}$ & Cathepsin L & $p$-value* & Cystatin C & $p$-value ${ }^{*}$ \\
\hline Serous & 41 & & 0.031 & & 0.131 & & 0.001 \\
\hline Serous cystadenoma & $29(71)$ & $12(1-269)^{\mathrm{a}}$ & & $31(9-121)$ & & $572(131-1,780)^{\mathrm{b}}$ & \\
\hline Serous borderline tumor & $2(5)$ & $14(8-21)$ & & $28(18-38)$ & & $1,449(1,125-1,772)$ & \\
\hline Serous cystadenocarcinoma & $10(24)$ & $65(3-269)^{\mathrm{a}}$ & & $61(8-163)$ & & $1,443(448-5,972)^{\mathrm{b}}$ & \\
\hline Mucinous & 35 & & 0.315 & & 0.017 & & 0.136 \\
\hline Mucinous cystadenoma & $21(60)$ & $16(1-227)$ & & $11(1-95)^{\mathrm{c}}$ & & $578(39-9,984)$ & \\
\hline Mucinous borderline tumor & $6(17)$ & $32(2-118)$ & & $75(8-166)$ & & $697(474-3,897)$ & \\
\hline Mucinous cystadenocarcinoma & $8(23)$ & $33(5-269)$ & & $31(13-187)^{\mathrm{c}}$ & & $959(399-9,984)$ & \\
\hline
\end{tabular}

* Statistical significance according to ANOVA

Student's $t$ test ${ }^{\mathrm{a}, \mathrm{b}, \mathrm{c}}$ of values normalized after $\operatorname{Ln}$ transformation

${ }^{\mathrm{a}} p=0.010$ for serous cystadenoma compared to serous cystadenocarcinoma

${ }^{\mathrm{b}} p=0.001$ for serous cystadenoma compared to serous cystadenocarcinoma

c $p=0.035$ for mucinous cystadenoma compared to mucinous cystadenocarcinoma

tumors $(n=41)$, CF levels of CatB were significantly higher for patients with malignant tumors compared to patients with benign tumors $(p=0.010)$, whereas CatL levels did not differ significantly within this histological subgroup. In contrast, in mucinous tumors $(n=35)$, CF levels of CatL were significantly higher for patients with malignant tumors compared to patients with benign tumors $(p=0.035)$, whereas CatB levels did not differ within this histological subgroup. CF levels of CysC only differed between patients with malignant and benign tumors in the subgroup of serous tumors $(p=0.001)$.

Parameters of aggressiveness of the tumor

Table 3 shows clinicopathological parameters of tumor aggressiveness and median (range) concentrations of CatB, $\mathrm{CatL}$ and $\mathrm{CysC}$ of the 26 patients with EOC. Since no differences in $\mathrm{CF}$ levels of $\mathrm{CatB}$, Cat $\mathrm{L}$ and $\mathrm{CysC}$ were found between serous, mucinous and endometrioid cystadenocarcinomas (Table 1), data of patients with EOC were merged. When patients were grouped according to FIGO stage of disease, tumor grading and presence of ascites, no significant differences in CF levels of CatB, CatL and/or $\mathrm{Cys} C$ were found.

Correlation between cathepsins and cystatin C

Figure 2 shows the scatter plots of the correlation between Cathepsins and Cystatin C for patients with serous (Fig. 2a and $2 \mathrm{~b} ; n=41$ ) and mucinous (Fig. 2c; $n=35$ ) tumors. For serous tumors, $\mathrm{CF}$ levels of $\mathrm{CysC}$ and $\mathrm{CatB}$ were significantly correlated ( $p<0.001, R=0.652$, Fig. $2 \mathrm{a}$ ). The correlation between $\mathrm{CF}$ levels of $\mathrm{Cys} \mathrm{C}$ and $\mathrm{CatB}$ was highest for the malignant group $(p<0.001, R=0.921)$. For mucinous tumors, $\mathrm{CF}$ levels of CysC and CatB did not show any relationship ( $p=0.923$, figure not shown). Correlations between $\mathrm{CF}$ levels of CysC and CatL were significant for both serous and mucinous tumors $(p=0.001, R=0.491$, Fig. $2 \mathrm{~b}$ and $p=0.003 ; R=0.481$, Fig. $2 \mathrm{c}$, respectively). For mucinous tumors, the correlation between CF levels of CysC and CatL was highest for the benign group $(p<0.001, R=0.665)$.

\section{Discussion}

In this study, we examined ovarian $\mathrm{CF}$ levels of $\mathrm{CatB}$, CatL and their extracellular inhibitor CysC. In benign tumors, remarkable differences in $\mathrm{CF}$ levels of cathepsins $\mathrm{B}$ and $\mathrm{L}$ were found between different histopathological subtypes. For serous tumors, the levels of $\mathrm{CatB}$ and CysC were significantly higher in the $\mathrm{CF}$ of patients with $\mathrm{EOC}$ compared to $\mathrm{CF}$ levels of patients with benign ovarian tumors. For mucinous tumors, the levels of CatL were significantly higher in the CF of patients with EOC compared to CF levels of patients with benign ovarian tumors. Furthermore, correlations between cyst fluid levels of CatB and CysC and between CatL and CysC were found. Finally, we investigated the relationship between the levels of $\mathrm{CatB}$, CatL and $\mathrm{CysC}$ and parameters of tumor aggressiveness of EOC, but could not find significant differences between the subgroups of patients.

We are the first to describe that $\mathrm{CF}$ of ovarian tumors contains considerable amounts of $\mathrm{CatB}$, CatL and CysC. In ascitic fluid of patients with ovarian cancer, Lah et al. (1992) found CatB and a large pool of free CysC. We showed that $\mathrm{CF}$ levels of these enzymes were higher in ovarian cancer patients compared to patients with benign 


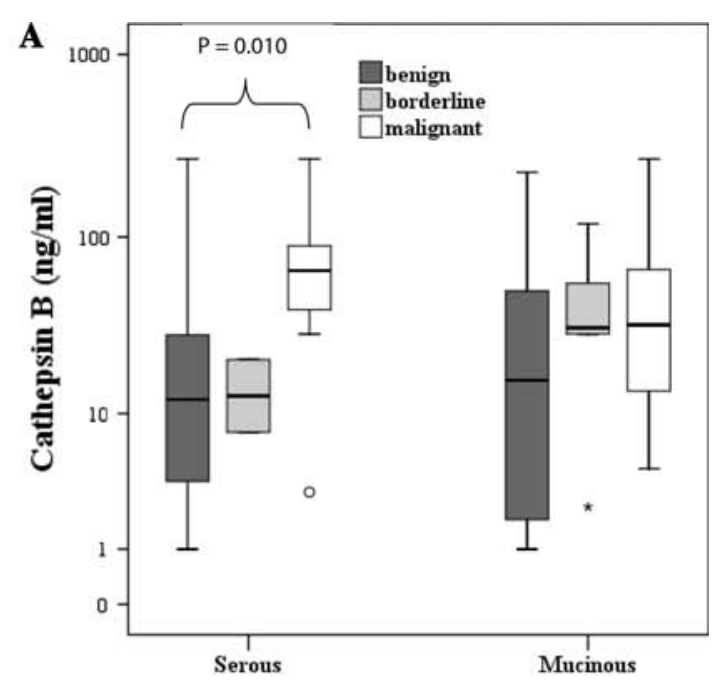

Histological subtype

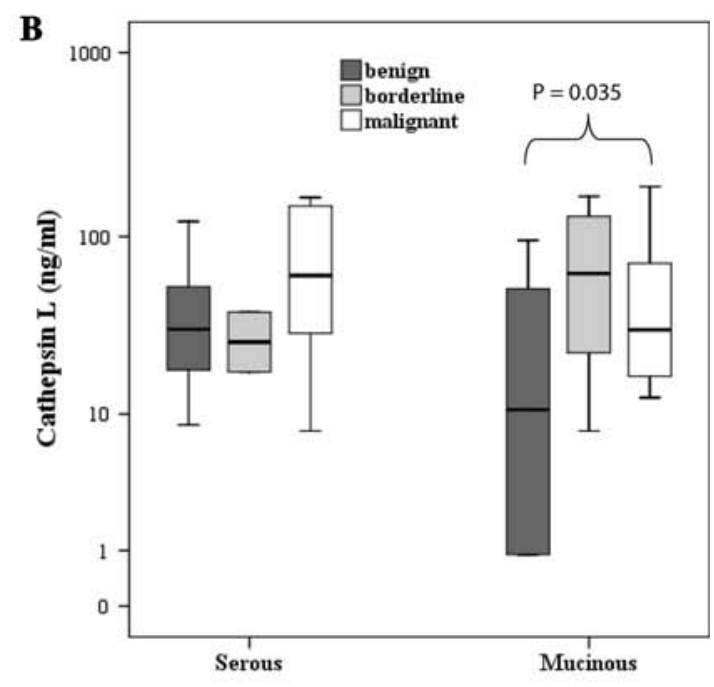

Histological subtype

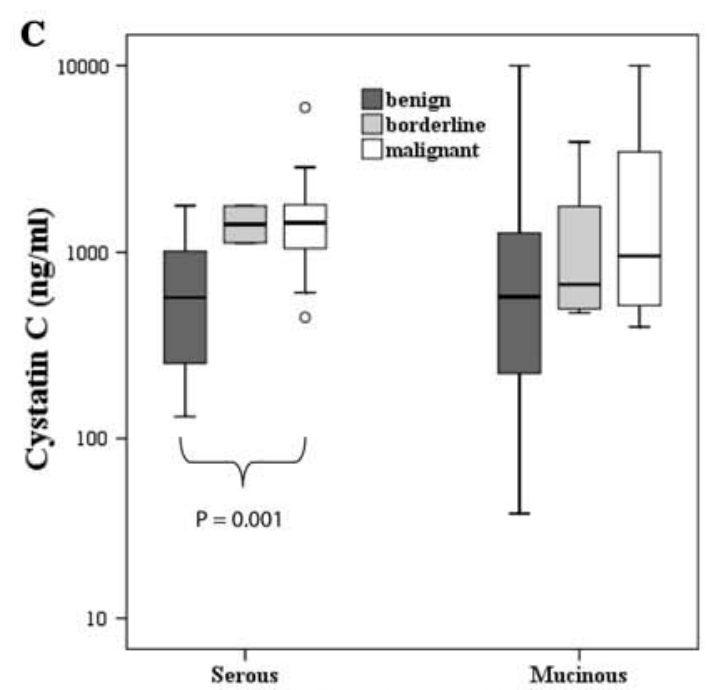

Histological subtype
4 Fig. 1 Box plots of the (a) cathepsin B and (b) cathepsin L, and (c) cystatin C ( $\mathrm{ng} / \mathrm{ml})$ concentrations (logarithmic scale) in ovarian cyst fluid for patients with malignant, borderline and benign ovarian tumors, clustered by histological subtype (serous and mucinous tumors). Statistical significance above the subfigures are according to ANOVA $(P<0.05)$ with Tukey's HSD post hoc tests of values normalized after Ln transformation

ovarian tumors. Serum studies in patients with other types of cancer have demonstrated similar results, as levels of CatB, CatL and CysC were found to be higher in serum of patients with cancer compared to serum levels of patients with benign tumors or healthy controls (Gabrijelcic et al. 1992; Kos et al. 1997, 1998; Leto et al. 1997a, b; Strojan et al. 2001; Zore et al. 2001; Yano et al. 2001; Makarewicz et al. 1995; Miyake et al. 2004; Strojan et al. 2004; Mulaomerovic et al. 2007). In serum of patients with ovarian cancer, Warwas et al. (1997) found higher levels of CatB compared to the levels in patients with benign ovarian tumors. On the other hand, Nishikawa et al. (2004) could not observe a significant difference in serum CatB level between patients with benign ovarian tumors and ovarian cancer, although they did find a significantly higher level of $\mathrm{CysC}$ in the serum of patients with ovarian cancer. Only one study has been published investigating CatL in the serum of patients with ovarian tumors (Nishida et al. 1995). The authors found that the serum levels of CatL were elevated in $8 / 10$ patients with ovarian cancer compared to $2 / 10$ patients with benign ovarian tumors.

The mean age of patients with ovarian cancer is known to be significantly higher than that of patients with benign ovarian tumors (Merino and Jaffe 1993). This was also found in our study group. This might confound our results, as there is definite evidence that renal function decreases with age (Lindeman and Goldman 1986), which results in a reduced glomerular filtration rate in older people. CysC is known to be almost completely reabsorbed and catabolized in the proximal renal tubular cells (Stabuc et al. 2000). This might explain the higher serum levels of CysC that were reported earlier in patients with ovarian cancer compared to that in patients with benign ovarian tumors. However, we found no correlation in $\mathrm{CF}$ between age and CysC level within the subgroups of patients. In addition, it is unclear whether CysC levels in ovarian $\mathrm{CF}$, in contrast to serum, are influenced by renal function. Finally, the group of patients with borderline tumors was of similar age as the patients with benign tumors, yet exhibited CatB, CatL and CysC levels similar to those of patients with ovarian cancer. The level of CysC might be changed also due to pathological renal failures, changing the glomerular filtration rate in cancer patients (Stabuc et al. 2000). However, patients included in this study, did not exhibit any impaired kidney 

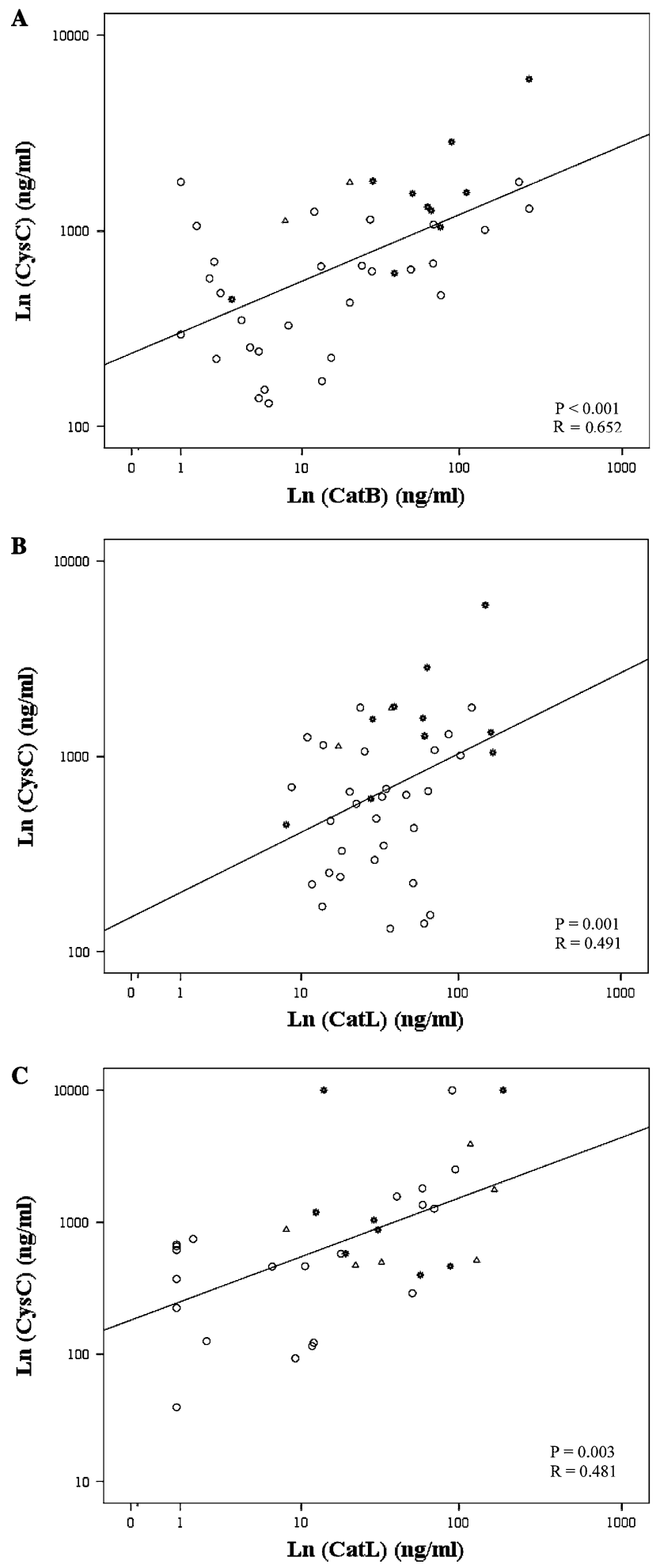

Fig. 2 Scatter plots of Ln-transformed values of cyst fluid cystatin C with cathepsin B (a) and cathepsin L (b) for patients with serous tumors $(n=41)$ and cystatin $\mathrm{C}$ with cathepsin $\mathrm{L}(\mathbf{c})$ for patients with mucinous tumors $(n=35)$. Statistical significance $(p<0.05)$ and correlation coefficient $(R)$ according to Pearson's correlation test. Asterisks malignant; open triangles borderline, open circles benign function. Therefore, we believe that the results of CysC in this study have not been biased by age-related or pathological changes of kidney function.

Remarkably, CatB and CysC CF levels were significantly higher in malignant serous tumors compared to benign serous tumors, whereas CatL CF levels were significantly higher in malignant mucinous tumors compared to benign mucinous tumors. This finding contributes to the theory that malignant serous and mucinous ovarian tumors are different diseases, with subtype-specific biomarker profiles (Heinzelmann-Schwarz et al. 2006; Kobel et al. 2008; Cloven et al. 2004). In addition, a significant correlation between CatB and CysC was found for patients with serous tumors. Moreover, the correlation of $\mathrm{CatB}$ and $\mathrm{Cys} \mathrm{C}$ was extremely strong in the group of patients with malignant serous tumors $(R=0.921)$, which might indicate that an increase of CF CatB in malignant tumors was balanced by a corresponding increase in $\mathrm{CF}$ CysC level. This finding could be interesting, as it was proposed in previous studies that a relative imbalance between $\mathrm{CatB}$ and $\mathrm{Cys} C$ in patients with cancer might contribute to the invasion and metastasis of tumor cells (Kos et al. 2000; Nishikawa et al. 2004; Lah et al. 2000).

Although not significant, median CF levels of CatB and CatL were up to seven times higher in cystic endometriosis compared to the other histopathological subtypes, and levels were comparable to CF levels of ovarian cancer patients with unfavorable tumor characteristics. Noteworthy is that CysC did not differ between cystic endometriosis and other benign subtypes, which might suggest a different balance between $\mathrm{CatB}$ and $\mathrm{Cys} \mathrm{C}$ in endometriosis cysts compared to the other histological subtypes. This might be explained by the continuous breakdown of components of blood and stromal tissue in endometriosis without a malignant invasion or metastasis. However, it is now well established that endometriosis does have neoplastic potential and it has been associated with the development of endometrioid and clear-cell ovarian carcinoma (Feeley and Wells 2001; Wells 2004).

Since ovarian cancer tissue is highly heterogeneous, multiple biopsies are necessary for a careful examination (Hogdall et al. 2007; Chauhan et al. 2007). This means that quantitation of cathepsins and cystatins in biological fluids of ovarian cancer patients has several clinical advantages over measurements in ovarian cancer tissue. We found that the levels of cathepsins and $\mathrm{CysC}$ in ovarian $\mathrm{CF}$ were much higher than that in serum of cancer patients, as reported in literature. More specifically, up to six times more CatB was found in $\mathrm{CF}$ than in the serum of patients with colorectal carcinoma (Kos et al. 1998) and melanoma (Kos et al. 1997), up to ten times more CatL was found in ovarian CF 
Table 3 Median levels (range) of cathepsin B, L and cystatin C (ng/ml) in malignant ovarian tumor cyst fluid $(n=26)$ according to clinicopathological parameters for aggressiveness of the tumor

\begin{tabular}{|c|c|c|c|c|c|c|c|}
\hline & $N(\%)$ & Cathepsin B & $p$-value & Cathepsin L & $p$-value & Cystatin C & $p$-value \\
\hline FIGO stage & & & $0.613^{\mathrm{a}}$ & & $0.984^{\mathrm{a}}$ & & $0.406^{\mathrm{a}}$ \\
\hline Ia-IIa & $13(50)$ & $48(28-93)$ & & $40(29-78)$ & & $1,040(594-2,355)$ & \\
\hline$\geq \mathrm{IIb}$ & $12(46)$ & $72(16-109)$ & & $63(18-125)$ & & $1,233(1,055-1,500)$ & \\
\hline Unknown & $1(4)$ & & & & & & \\
\hline Tumor grade & & & $0.554^{\mathrm{b}}$ & & $0.595^{\mathrm{b}}$ & & $0.535^{\mathrm{b}}$ \\
\hline 1 & $6(23)$ & $38(10-269)$ & & $31(14-187)$ & & $959(465-9,984)$ & \\
\hline 2 & $5(19)$ & $71(38-269)$ & & $62(38-163)$ & & $1,076(399-1,502)$ & \\
\hline 3 & $13(50)$ & $64(3-11)$ & & $64(8-181)$ & & $1,324(448-2,908)$ & \\
\hline Unknown & $2(8)$ & & & & & & \\
\hline Ascites & & & $0.632^{\mathrm{a}}$ & & $0.379^{\mathrm{a}}$ & & $0.569^{\mathrm{a}}$ \\
\hline Yes & $11(42)$ & $76(5-269)$ & & $64(13-181)$ & & $1,330(1,048-2,908)$ & \\
\hline No & $12(46)$ & $55(10-269)$ & & $48(14-187)$ & & $970(399-9,984)$ & \\
\hline Unknown & $3(12)$ & & & & & & \\
\hline
\end{tabular}

Statistical significance according to

a Student's $t$ test

b ANOVA of values normalized after Ln transformation

than in serum of patients with ovarian cancer (Nishida et al. 1995), and up to eight times more CysC was found in ovarian CF than in the serum of patients with ovarian cancer (Nishikawa et al. 2004). This might indicate that CatB, $\mathrm{CatL}$ as well as CysC are released from tumor cells into the $\mathrm{CF}$ to a greater extent than the release of these compounds into the serum of cancer patients. In this way, $\mathrm{CF}$ values may give a better reflection of local changes of the ovarian tumor than levels of cathepsins and its inhibitors in serum, which also might be affected by the systemic response on malignant disease and circadian variations (Cimerman et al. 1999, 2000). From a clinical perspective, preoperative aspiration of ovarian cyst fluid might cause iatrogenic metastasis of tumor cells, and ovarian cyst fluid can only be used as a source of biomarkers for predicting prognosis and response to therapy. We, therefore, investigated the relationship between clinicopathological parameters that correspond with tumor aggressiveness and the levels of CatB, $\mathrm{CatL}$ and $\mathrm{Cys} C$ in patients with ovarian cancer. However, when patients were grouped according to FIGO stage of disease, tumor grading and presence of ascites, no significant differences in $\mathrm{CF}$ levels of CatB, CatL and/or CysC were found. This could be caused by the relatively small size of cyst fluid samples from malignant tumors in the present study.

\section{Conclusions}

$\mathrm{CF}$ levels of $\mathrm{CatB}$, and its endogenous inhibitor $\mathrm{CysC}$, were significantly higher in serous EOC compared to benign serous ovarian tumors, whereas $\mathrm{CF}$ levels of Cat L were significantly higher in mucinous EOC compared to benign mucinous ovarian tumors. The correlation between CatB and $\mathrm{Cys} C$ was extremely strong for patients with serous EOC, which might indicate that an increase in CatB of these patients is balanced by a corresponding increase in CysC level. We feel that further studies with a larger series of samples from malignant tumors are needed to explore the possible prognostic value of cathepsins and $\mathrm{CysC}$ in ovarian $\mathrm{CF}$.

Open Access This article is distributed under the terms of the Creative Commons Attribution Noncommercial License which permits any noncommercial use, distribution, and reproduction in any medium, provided the original author(s) and source are credited.

\section{References}

Aznavoorian S, Murphy AN, Stetler-Stevenson WG, Liotta LA (1993) Molecular aspects of tumor cell invasion and metastasis. Cancer 71(4): 1368-1383

Bhuvarahamurthy V, Govindasamy S (1995) Extracellular matrix components and proteolytic enzymes in uterine cervical carcinoma. Mol Cell Biochem 144(1):35-43

Capo-Chichi CD, Smith ER, Yang DH, Roland IH, Vanderveer L, Cohen C, Hamilton TC, Godwin AK, Xu XX (2002) Dynamic alterations of the extracellular environment of ovarian surface epithelial cells in premalignant transformation, tumorigenicity, and metastasis. Cancer 95(8):1802-1815

Chauhan SC, Vinayek N, Maher DM, Bell MC, Dunham KA, Koch MD, Lio Y, Jaggi M (2007) Combined staining of TAG-72, MUC1, and CA125 improves labeling sensitivity in ovarian cancer: antigens for multi-targeted antibody-guided therapy. J Histochem Cytochem 55(8):867-875 
Cimerman N, Brguljan PM, Krasovec M, Suskovic S, Kos J (1999) Circadian characteristics of cathepsins B, H, L, and stefins A and $\mathrm{B}$, potential markers for disease, in normal sera. Clin Chim Acta 282(1-2):211-218

Cimerman N, Brguljan PM, Krasovec M, Suskovic S, Kos J (2000) Twenty-four hour variations of cystatin $\mathrm{C}$ and total cysteine proteinase inhibitory activity in sera from healthy subjects. Clin Chim Acta 291(1):89-95

Cloven NG, Kyshtoobayeva A, Burger RA, Yu IR, Fruehauf JP (2004) In vitro chemoresistance and biomarker profiles are unique for histologic subtypes of epithelial ovarian cancer. Gynecol Oncol 92(1):160-166

Colombo N, Van GT, Parma G, Amant F, Gatta G, Sessa C, Vergote I (2006) Ovarian cancer. Crit Rev Oncol Hematol 60(2):159-179

Duffy MJ (1996) Proteases as prognostic markers in cancer. Clin Cancer Res 2(4):613-618

Feeley KM, Wells M (2001) Precursor lesions of ovarian epithelial malignancy. Histopathology 38(2):87-95

Gabrijelcic D, Svetic B, Spaic D, Skrk J, Budihna M, Dolenc I, Popovic T, Cotic V, Turk V (1992) Cathepsins B, H and L in human breast carcinoma. Eur J Clin Chem Clin Biochem 30(2):69-74

Heinzelmann-Schwarz VA, Gardiner-Garden M, Henshall SM, Scurry JP, Scolyer RA, Smith AN, Bali A, Vanden BP, Baron-Hay S, Scott C, Fink D (2006) A distinct molecular profile associated with mucinous epithelial ovarian cancer. Br J Cancer 94(6):904-913

Hogdall EV, Christensen L, Kjaer SK, Blaakaer J, Kjaerbye-Thygesen A, Gayther S, Jacobs IJ, Hogdall CK (2007) CA125 expression pattern, prognosis and correlation with serum CA125 in ovarian tumor patients. From The Danish "MALOVA" Ovarian Cancer Study. Gynecol Oncol 104(3):508-515

Kobel M, Kalloger SE, Boyd N, McKinney S, Mehl E, Palmer C, Leung S, Bowen NJ, Ionescu DN, Rajput A, Prentice LM (2008) Ovarian carcinoma subtypes are different diseases: implications for biomarker studies. PLoS Med 5(12):e232

Kos J, Smid A, Krasovec M, Svetic B, Lenarcic B, Vrhovec I, Skrk J, Turk V (1995) Lysosomal proteases cathepsins D, B, H, L and their inhibitors stefins $\mathrm{A}$ and $\mathrm{B}$ in head and neck cancer. Biol Chem Hoppe Seyler 376(7):401-405

Kos J, Stabuc B, Schweiger A, Krasovec M, Cimerman N, KopitarJerala N, Vrhovec I (1997) Cathepsins B, H, and L and their inhibitors stefin A and cystatin $\mathrm{C}$ in sera of melanoma patients. Clin Cancer Res 3(10): 1815-1822

Kos J, Nielsen HJ, Krasovec M, Christensen IJ, Cimerman N, Stephens RW, Brunner N (1998) Prognostic values of cathepsin B and carcinoembryonic antigen in sera of patients with colorectal cancer. Clin Cancer Res 4(6):1511-1516

Kos J, Werle B, Lah T, Brunner N (2000) Cysteine proteinases and their inhibitors in extracellular fluids: markers for diagnosis and prognosis in cancer. Int J Biol Markers 15(1):84-89

Lah TT, Kokalj-Kunovar M, Kastelic L, Babnik J, Stolfa A, Rainer S, Turk V (1992) Cystatins and stefins in ascites fluid from ovarian carcinoma. Cancer Lett 61(3):243-253

Lah TT, Strojnik T, Levicar N, Bervar A, Zajc I, Pilkington G, Kos J (2000) Clinical and experimental studies of cysteine cathepsins and their inhibitors in human brain tumors. Int $\mathrm{J}$ Biol Markers 15(1):90-93

Landen CN Jr, Birrer MJ, Sood AK (2008) Early events in the pathogenesis of epithelial ovarian cancer. J Clin Oncol 26(6):995-1005

Leto G, Tumminello FM, Pizzolanti G, Montalto G, Soresi M, Gebbia N (1997a) Lysosomal cathepsins B and L and Stefin A blood levels in patients with hepatocellular carcinoma and/or liver cirrhosis: potential clinical implications. Oncology 54(1):79-83

Leto G, Tumminello FM, Pizzolanti G, Montalto G, Soresi M, Carroccio A, Ippolito S, Gebbia N (1997b) Lysosomal aspartic and cysteine proteinases serum levels in patients with pancreatic cancer or pancreatitis. Pancreas 14(1):22-27
Lindeman RD, Goldman R (1986) Anatomic and physiologic age changes in the kidney. Exp Gerontol 21(4-5):379-406

Liotta LA, Tryggvason K, Garbisa S, Hart I, Foltz CM, Shafie S (1980) Metastatic potential correlates with enzymatic degradation of basement membrane collagen. Nature 284(5751):67-68

Makarewicz R, Drewa G, Szymanski W, Skonieczna-Makarewicz I (1995) Cathepsin B in predicting the extent of the cervix carcinoma. Neoplasma 42(1):21-24

Merino MJ, Jaffe G (1993) Age contrast in ovarian pathology. Cancer 71(Suppl 2):537-544

Miyake H, Hara I, Eto H (2004) Serum level of cathepsin B and its density in men with prostate cancer as novel markers of disease progression. Anticancer Res 24(4):2573-2577

Mulaomerovic A, Halilbasic A, Cickusic E, Zavasnik-Bergant T, Begic L, Kos J (2007) Cystatin C as a potential marker for relapse in patients with non-Hodgkin B-cell lymphoma. Cancer Lett 248(2):192-197

Nishida Y, Kohno K, Kawamata T, Morimitsu K, Kuwano M, Miyakawa I (1995) Increased cathepsin L levels in serum in some patients with ovarian cancer: comparison with CA125 and CA724. Gynecol Oncol 56(3):357-361

Nishikawa H, Ozaki Y, Nakanishi T, Blomgren K, Tada T, Arakawa A, Suzumori K (2004) The role of cathepsin B and cystatin C in the mechanisms of invasion by ovarian cancer. Gynecol Oncol 92(3):881-886

Pecorelli S, Benedet JL, Creasman WT, Shepherd JH (1999) FIGO staging of gynecologic cancer. 1994-1997 FIGO Committee on Gynecologic Oncology. International Federation of Gynecology and Obstetrics. Int J Gynaecol Obstet 65(3):243-249

Premzl A, Turk V, Kos J (2006) Intracellular proteolytic activity of cathepsin B is associated with capillary-like tube formation by endothelial cells in vitro. J Cell Biochem 97(6):1230-1240

Schwartz MK (1995) Tissue cathepsins as tumor markers. Clin Chim Acta 237(1-2):67-78

Scully RE (1995) Pathology of ovarian cancer precursors. J Cell Biochem Suppl 23:208-218

Servov SF, Scully RE, Sobin LH (1973) International histologic classification of tumors. No. 9: histologic typing of ovarian tumors. World Health Organization, Geneva, 1973

Sloane BF, Moin K, Krepela E, Rozhin J (1990) Cathepsin B and its endogenous inhibitors: the role in tumor malignancy. Cancer Metastasis Rev 9(4):333-352

Stabuc B, Vrhovec L, Stabuc-Silih M, Cizej TE (2000) Improved prediction of decreased creatinine clearance by serum cystatin C: use in cancer patients before and during chemotherapy. Clin Chem 46(2): 193-197

Strojan P, Budihna M, Smid L, Svetic B, Vrhovec I, Skrk J (2001) Cathepsin B and L and stefin A and B levels as serum tumor markers in squamous cell carcinoma of the head and neck. Neoplasma 48(1):66-71

Strojan P, Svetic B, Smid L, Kos J (2004) Serum cystatin C in patients with head and neck carcinoma. Clin Chim Acta 344(1-2):155-161

Turk B, Turk D, Turk V (2000) Lysosomal cysteine proteases: more than scavengers. Biochim Biophys Acta 1477(1-2):98-111

Warwas M, Haczynska H, Gerber J, Nowak M (1997) Cathepsin B-like activity as a serum tumour marker in ovarian carcinoma. Eur J Clin Chem Clin Biochem 35(4):301-304

Wells M (2004) Recent advances in endometriosis with emphasis on pathogenesis, molecular pathology, and neoplastic transformation. Int J Gynecol Pathol 23(4):316-320

Yano M, Hirai K, Naito Z, Yokoyama M, Ishiwata T, Shiraki Y, Inokuchi M, Asano G (2001) Expression of cathepsin B and cystatin $\mathrm{C}$ in human breast cancer. Surg Today 31(5):385-389

Zore I, Krasovec M, Cimerman N, Kuhelj R, Werle B, Nielsen HJ, Brunner N, Kos J (2001) Cathepsin B/cystatin C complex levels in sera from patients with lung and colorectal cancer. Biol Chem 382(5):805-810 